\title{
Note sur l'acquisition des voyelles du français par les apprenants chinois avec la L2 anglais
}

\author{
Xiaoliang $\mathrm{LUO}^{1, *}$ et Qianwen $\mathrm{ZHAO}^{2}$ \\ ${ }^{1}$ Laboratoire Ligérien de Linguistique, UMR7270, Université d'Orléans \\ ${ }^{2}$ Institut d'Etudes Européennes, Université Sorbonne Nouvelle
}

\begin{abstract}
Résumé. Les apprenants chinois ont souvent de grandes difficultés de prononciation lorsqu'ils apprennent le français. Ces difficultés ne sont pas seulement liées à leur langue maternelle : l'anglais joue également un rôle important dû au système éducatif chinois. Croyant que l'anglais serait plus proche du français, certains apprenants chinois cherchent des prononciations similaires dans la langue anglaise alors que certains sons français existent phonétiquement en chinois. Dans cet article, nous nous concentrons sur l'acquisition des voyelles du français et essayons de déterminer les influences de la L1 et de la L2 respectivement, en nous basant sur un corpus constitué d'enregistrements des apprenants ayant des profils homogènes.
\end{abstract}

\begin{abstract}
Chinese learners of French with English as L2 in the learning of vowel system. Chinese learners often face great pronunciation difficulties when learning French. These difficulties are not only caused by L1 Chinese, but also strongly linked to L2 English, after years of learning from primary school to high school. Believing that English is closer to French, Chinese learners often look for similar pronunciations in English while some French sounds exist phonetically in Chinese. In this paper we focus on their acquisition of French vowels and try to identify the influences of L1 and L2 respectively, based on a corpus with homogeneous learner profiles in uncontrolled speech.
\end{abstract}

\section{INTRODUCTION}

Beaucoup d'études ont été effectuées sur l'acquisition de la L3, mais des analyses fines de l'acquisition du français comme L3 par des apprenants chinois avec la L2 anglais restent peu nombreuses, en particulier dans le contexte actuel où la Chine compte un grand nombre d'apprenants de français. Plus spécifiquement, l'acquisition de la phonétique par ce public reste quelque peu absente dans l'état de l'art.

*xiaoliang.luo@univ-orleans.fr 
L'anglais, bien que faisant partie de la famille indo-européenne et partageant un système d'écriture semblable à celui du français aux yeux des apprenants chinois, n'est pas toujours un facteur facilitant l'apprentissage du français.

En acquisition de la L3, le transfert est souvent double en lexique et en syntaxe. A titre d'exemple, en lexique, Wlosowicz 2010 montre l'efficacité de l'apprentissage lors du transfert positif tout en soulignant qu'en cas de différences entre la L2 et la L3, «l'interférence et le transfert négatifs sont particulièrement visibles ». En syntaxe, Bardel (2006) conduit à la conclusion que « la connaissance des structures syntaxiques d'une L2 peut être transférée à une L3, ce qui facilite de manière significative l'acquisition de structures similaires », mais «l'acquisition de la négation préverbale ne va pas de soi pour les apprenants ayant connaissance de langues sources à négation postverbale ».

En phonétique, cela paraît différent, ce qui est en lexique transfert positif pourrait donner lieu au contraire à des interférences et transferts négatifs. Par exemple, la ressemblance entre le mot anglais « university » et le mot français " université » facilite clairement l'apprentissage du vocabulaire, ayant un effet de transfert positif, mais cette ressemblance pose un obstacle supplémentaire pour l'apprenant ayant l'anglais comme L2, car il a plus de chance d'imiter la prononciation anglaise, qu'il connaît déjà, pour prononcer le mot français. C'est pourquoi nous pensons devoir accorder une attention particulière à la phonétique dans l'acquisition de la L3.

Quatre types de littérature concernent notre problématique : 1) l'influence de la L2 sur la L3 en général ; 2) l'acquisition de la phonétique du français par les apprenants chinois ; 3) l'acquisition du français comme L3 par les apprenants chinois ayant l'anglais comme L2 ; 4) l'influence de la L2 anglais sur la prononciation de la L3 français.

La première catégorie, c'est-à-dire la littérature sur l'influence de la L2 sur l'apprentissage de la L3 (Hammarberg 2001 ; Gut 2010 ; Llama et als. 2010 entre autres) ne traite pas directement de l'étude de cas de l'acquisition du français en L3 par des apprenants chinois ayant l'anglais comme L2.

L'acquisition de la phonétique du français par les apprenants chinois a été abordée dans Martin (2002) ; Gao Y. \& Shi F. (2006); Landron et als. (2016) ; Huang Y. et als. (2018) ; Li J. et als. (2018) ; Rimpault (2018), mais l'influence de la L2 anglais n'est pas vraiment prise en compte en détail. Landron et als. (2016) détaillent de façon qualitative un certain nombre de prononciations typiques chez les sinophones, cependant, il n'y a pas de discussion sur si une prononciation est due à l'influence de L1 ou de L2.

Wang H. \& Ma J. (2005), Zhou X. (2006), Cuet (2008, 2009a, 2009b, 2011) s'intéressent à l'acquisition du français par des apprenants chinois ayant l'anglais comme L2 de façon générale et abordent surtout le lexique, la sémantique et la pragmatique mais non pas la phonétique.

Enfin, quelques réflexions sur cette question, telles que Wang M. (2008), Xu C. \& Zhang J. (2011), existent dans la littérature en chinois d'un point de vue didactique, mais il s'agit de petites notes plutôt que d'articles de recherche, d'une longueur comprise entre 2 et 4 pages, sans méthodes qualitatives ni quantitatives.

En conséquence, il nous paraît nécessaire d'aborder la problématique de façon minutieuse, en adoptant une méthode quantitative qui mènera à une analyse qualitative. Dans cet article, nous nous limitons au vocalisme du français.

Les locuteurs de chinois mandarin (désormais CM) ont souvent un dialecte ${ }^{\mathrm{i}}$. Nous sommes conscients que le dialecte natif pourrait avoir un impact non négligeable sur 
l'acquisition, mais nous ne considérerons pas son influence dans cette étude pour deux raisons.

Premièrement, les origines des 44 locuteurs se répartissent de façon aléatoire sur tout le territoire chinois, ce qui rend cette variable incontrôlable à ce stade (mais nous comptons la prendre en compte dans des études ultérieures).

Deuxièmement, avec la généralisation du $\mathrm{CM}$, les jeunes générations (qui constituent notre corpus) perdent rapidement leurs dialectes et leur influence ainsi diminue. Un des signes de cette perte est que dans la ville natale de l'un des auteurs, les lycéens échangent en CM même en dehors des heures de cours, ce qui n'était pas du tout le cas il y a deux décennies.

\section{CORPUS ET METHODE}

\subsection{Corpus}

Le corpus de cette recherche est constitué de 44 enregistrements réalisés avec le consentement de tous les participants en juin 2017. Toutes les personnes interrogées ont des profils homogènes : des étudiants natifs du $\mathrm{CM}$, ayant au moins dix ans d'expérience dans l'apprentissage de l'anglais. N'ayant jamais fait de français, ils se sont inscrits à des cours intensifs de français niveau débutant à Châteauroux de novembre 2016 à juin 2017. Le programme a duré 26 semaines, avec 20 heures de cours par semaine. A la fin de l'année universitaire, chaque étudiant devait tenir un entretien avec le professeur de phonétique pendant quelques minutes. C'est au cours de ces discours spontanés que les étudiants étaient enregistrés et évalués sur la prononciation. Initialement prévus à des fins pédagogiques, ces enregistrements n'ont pas été enregistrés dans des conditions optimales.

\subsection{Méthode}

La prononciation de chaque voyelle du français de chaque apprenant est comparée avec la prononciation cible correspondante. Les prononciations non standard sont ensuite classées en deux catégories : Les réalisations dominées par la L1 ou celles par la L2. Pour mettre en évidence les influences de la L1 et de la L2 respectivement en termes de prononciation, trois autres aspects susceptibles d'interférer sont exclus dans cet article : l'influence du système d'écriture, l'influence de la structure syllabique ainsi que l'inconstance du même apprenant. A cet égard, les discours spontanés enregistrés nous permettent de minimiser les influences des systèmes d'écritures, en particulier celui de l'anglais; seules les réalisations répétées d'un locuteur sont considérées comme pertinentes. L'influence de la structure syllabique en revanche nécessite un corpus contrôlé. Pour analyser de façon systématique l'impact de la position de la voyelle au sein du mot et de la syllabe, il nous paraît nécessaire de réaliser dans notre futur travail une liste de mots épuisant toutes les possibilités et les faire répéter aux sujets. Ainsi à ce stade nous pensons que le discours spontané de notre corpus n'est pas en mesure d'évaluer cet aspect. Les facteurs positionnels impliquent également des analyses phonologiques fines concernant les trois langues en termes de contraintes syllabiques, combinabilité, accentuation, sonorité, ainsi que la prise en compte des phénomènes comme épenthèse et élision, ce qui dépassent l'objectif de cet article. Néanmoins, cela ne nous exclut pas de commenter occasionnellement ces phénomènes quand leur influence est visible. 
Pour déterminer si une réalisation est dominée par la L1 ou la L2, des connaissances approfondies des systèmes vocaliques de toutes les trois langues concernées sont nécessaires.

\section{SYSTEMES VOCALIQUES}

\subsection{Système vocalique du chinois mandarin}

Dans cette section, nous présentons le système vocalique du CM, celui de l'anglais et du français étant familier pour les lecteurs.

Selon Duanmu (2007), le CM a un système à cinq voyelles comme suit :

(1) Système vocalique du CM

$\begin{array}{lll}\text { i } & \text { y } & \text { u } \\ & \text { e } & \\ & \text { a } & \end{array}$

La voyelle moyenne /e/ a quatre allophones : [e] antérieur non-arrondi, [o] postérieur et arrondi, $[\gamma]$ postérieur non-arriondi et le schwa [ə]. La realisation phonétique est prédictible selon la structure syllabique du CM composé de quatre constituants : l'initiale, la médiane, le noyau et la finale. Cette analyse syllabique existe depuis la tradition philologique de la Chine Antique et a été reformulé par Duanmu (2007) comme CGVX, où C pour l'attaque, $\mathrm{G}$ pour glide, $\mathrm{V}$ pour la voyelle principale et $\mathrm{X}$ pour soit la seconde partie d'une diphtongue ([i] ou [u]), soit la coda nasale ([n] ou [y]). Cf. aussi Pöchtrager \& Živanović (2010) pour une analyse détaillée de la structure syllabique du CM avec une approche plus explicative.

La combinaison de la voyelle principale et du glide i / u avant ou après elle donne les diphtongues (montantes et descendantes) bien formées en $\mathrm{CM}$ comme suit :

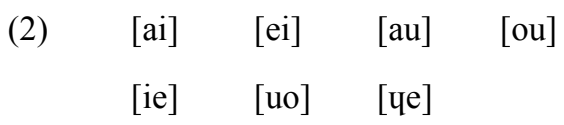

Selon Luo (2013), les nasales finales du CM font parties de l'Unité Porteuse de Ton (TBU) et doivent être considérées comme la seconde partie de la diphtongue. Cette vision date d'Antoine Meillet (1995) qui propose que des séquences VN peuvent être analysées comme des diphtongues. Les combinaisons possibles des diphtongues du type VN sont montrées dans (3) :

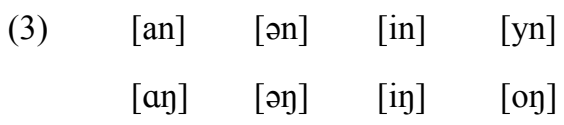


Le CM est une langue à tons ayant quatre tons lexicaux: plat, montant, descendant-montant et descendant. Landron et als. (2016) ne font pas mention à l'influence des tons sur la prononciation du français et selon nous il n'y a pas d'impact visible sur la prononciation des segments. Son influence est d'ordre prosodique : les sinophones ont une intonation très hachée en parlant français et rencontrent beaucoup de difficulté quand ils imitent une intonation lisse ayant comme portée la phrase. Toutefois, nous ne développerons pas cet aspect qui mérite une étude à part entière.

\subsection{Système vocalique du français}

Le français enseigné au CES Châteauroux est le français considéré comme standard. Etant bien conscients que la notion même de «français standard " peut être mise en cause et que la variation régionale et sociale est omniprésente au sein de la même communauté linguistique, nous adoptons le système vocalique du français, adapté de Léon (2007:114), montré dans (4) :

$\begin{array}{llll}\mathrm{i} & \mathrm{y} & & \mathrm{u} \\ \mathrm{e} & \varnothing & \partial & \mathrm{o} \text { o } \\ \varepsilon \tilde{\varepsilon} & \propto & & 0 \\ \mathrm{a} & & & \mathrm{a}^{\sim}\end{array}$

Le français, tel qu'il est décrit par Léon (2007), n'a pas de diphtongue et toutes les monophtongues sont tendues. Une révision mineure de (4) concerne le schwa, du point de vue phonologique, c'est un objet vide qui peut faire surface et alterne avec zéro, conditionné par la morphophonologie (cf. Scheer 1999 entre autres); du point de vue phonétique, ce schwa est traditionnellement décrit comme une voyelle neutre dont les trois formants sont à intervalle égale (cf. Landercy \& Renard 1977 entre autres). Cependant, selon les statistiques faites par un des auteurs de 2009 à 2015 au Département de SDL à l'Université d'Orléans auprès des locuteurs natifs majoritairement issus de la Région Centre-Val-de-Loire dans les groupes de TD en phonétique, aucune distinction, ni perceptive, ni articulatoire, est présente chez ce public jeune, entre le schwa et le [ø] antérieur arrondi et mi-haut. Bien sûr, nous ne prétendons pas élargir cette constatation sur tout le territoire français.

\subsection{Traits du système vocalique de l'anglais}

Il existe nombre de variétés de l'anglais. Dans le cas de cette étude, l'anglais qui nous intéresse doit être l'anglais acquis comme L2 par les sujets du corpus, c'est-à-dire l'anglais qui est enseigné en Chine. Mei (2003) souligne que dans l'enseignement élémentaire et secondaire, les élèves sont souvent exposés à la fois aux anglais britannique et américain. Selon He (2004), le marché de l'enseignement de l'anglais en Chine est partagé entre l'anglais britannique (majorité) et l'anglais américain (minorité).

Bien que les notions mêmes de l'anglais britannique et l'anglais américain peuvent être mises en cause, à savoir la question de norme et de variation, il est important de souligner seuls leurs traits communs qui auraient des impacts sur l'apprentissage du 
français et qui nous servirons pour déterminer l'influence de l'anglais dans la prononciation de nos sujets :

1) absences de certaines voyelles (notamment la série antérieure arrondie)

2) existence des diphtongues, absentes en français ;

3) série de voyelles relâchées, vs tendues en français ;

4) absences de voyelles nasales phonémiques

\section{REALISATIONS TYPIQUES DES VOYELLES DU FRANÇAIS}

Landron et als. (2016: 197) constatent un certain nombre de prononciations typiques des apprenants sinophones, qui concordent avec nos observations, mais cette étude n'épuise pas tout le système vocalique et ne cherche pas à identifier l'influence de la L1 et de la L2. Dans cette partie, nous allons recenser toutes les voyelles du français et essayerons de déterminer l'influence de chacune dans $\S 4$.

\subsection{Voyelles orales}

\subsubsection{Voyelles hautes}

La réalisation des voyelles hautes [i], $[\mathrm{y}]$ et $[\mathrm{u}]$ est marquée par l'influence de l'anglais sous deux formes différentes: tension et diphtongaison, alors que toutes les trois existent phonétiquement en CM. Au lieu de chercher des sons proches dans leur langue maternelle, les apprenants essayer de les trouver en anglais.

Pour [i] et [u], les étudiants prononcent systématiquement leurs versions relâchées : [i] est réalisé par 14 apprenants comme [I] relâché de l'anglais, comme dans " université »

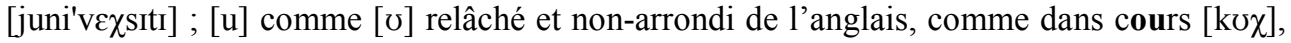

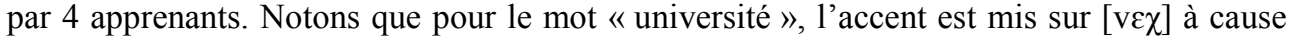
de la ressemblance au mot anglais, il s'agit clairement ici d'un transfert positif en lexique mais négatif en phonétique.

Quant à [y], il est réalisé comme la diphthongue [ju:] de l'anglais par 5 apprenants, comme dans le mot « bus » [bju:s].

\subsubsection{Voyelles mi-hautes}

La réalisation des voyelles mi-hautes [e], [o] et [ø] se divisent aussi en deux stratégies différentes.

Le [e] et le [o] sont réalisés de façon instable qui forment des diphtongues, ce qui confirme Landron et als. (2016). [e] est réalisé comme [eI] par 6 apprenants comme dans « spécialité » [spersjali'ter], alors que [o] est réalisé comme [ou] par 13 apprenants, par exemple dans "chose» [6ouz], ou comme [au] par 3 apprenants, par exemple «au cinéma » [au'si:nema]. Une observation intéressante est que [e] a tendance de rester [e] et ne pas se diphtonguer lorsqu'il est atone, c'est le cas de "cinéma » réalisé comme ['si:nema]. Lorsqu'il est sous accent ou réalisé avec de l'énergie, il a tendance à devenir la 
diphtongue [er], comme dans «spécialité » [spersjali'teI], où [ter] est sous accent et [sper] sous une sorte d'accent « secondaire ».

Pour la voyelle antérieure arrondie [ø], il n’y a pas de diphtongaison, cette voyelle ne ressemble à aucune voyelle anglaise et les apprenants cherchent des monophtongues les plus proches en CM. [ø] est réalisé comme [ $\gamma$ ] par 11 apprenants : « deuxième » ['tr:zjem], et comme $[\mathrm{u}]$ par 9 apprenants : «peu » $[\mathrm{pu}]^{\mathrm{ii}}$. Cela concorde avec Landron et als. (2016 : 197).

Une question à laquelle il faut répondre est la suivante : $[\gamma]$ est la version postérieure non-arrondie ayant la même aperture que $[\varnothing]$, alors que $[\mathrm{u}]$ est la version haute et postérieure ayant le même arrondissement. Pourquoi les apprenants abandonnent-ils [o] comme candidat potentiel dans notre corpus, bien que [o] soit plus proche de [ø], étant sa version postérieure ayant à la fois la même aperture et le même arrondissement ? Cette stratégie pourrait être expliquée par la phonotactique du CM. En CM, [o] est un allophone de la voyelle moyenne qui n'apparaît jamais isolément, contrairement à [r] qui le peut. Des

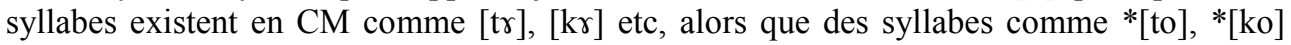
sont mal formées. [o] se trouve uniquement dans des diphtongues [uo], [ou] et [op], par assimilation de $[\mathrm{u}]$ postérieur et [y] vélaire. Ainsi, bien que [o] existe dans l'inventaire des sons du CM, la combinaison consonne $+[\mathrm{o}]$ est inexistante. Cela montre la nécessité de prendre en compte la combinabilité des segments dans des études ultérieures.

\subsubsection{Voyelles mi-basses}

Parallèlement, pour les trois voyelles mi-basses $[\varepsilon]$, [œ] et [॰], les apprenants adoptent des stratégies similaires à celles des voyelles mi-hautes.

Pour $[\varepsilon]$ et [o], les apprenants les adaptent comme des diphtongues. [ $\varepsilon]$ est réalisé par 28 apprenants comme la diphtongue [ar]: "mère " [mar $\chi$ ] ; 2 des apprenants remplacent $[\varepsilon]$ par $[æ]$ de l'anglais qui est plus bas : « mais » $[\mathrm{m} \mathfrak{e}]$.

Quant à [0], il est réalisé par 10 apprenants comme diphtongue [av]: «nous sommes » [nusaom].

[œ], de la même façon que [ø], pose un problème majeur aux apprenants, 17 parmi

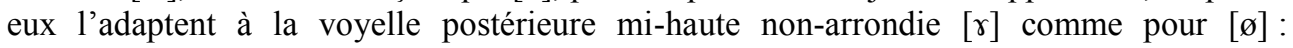
« heure » $[r \chi]$, ce qui correspond aux observations de Landron et als. (2016:197).

\subsubsection{Voyelle basse et schwa}

La voyelle basse [a] existe en CM, mais pour une nouvelle fois, 9 apprenants sur 44 sont sourds à cette voyelle qui existe (phonétiquement et phonologiquement) dans leur langue maternelle, au profit d'un [æ] anglais, par exemple : « Annecy » ['?ænsi]

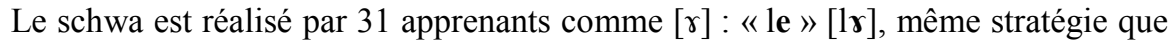
celle de $[\varnothing]$ et de [œ]. Comme mentionné plus haut, le schwa est phonétiquement confondu avec [ø] par les jeunes natifs en Région Centre-Val-de-Loire, cela confirmant cette difficulté majeure des sinophones pour prononcer les voyelles moyennes antérieures arrondies.

\subsection{Voyelles nasales}


Les voyelles nasales n'existent pas phonologiquement en CM même si les séquences VN en position non-finale du mot deviennent automatiquement des voyelles nasales phonétiquement (cf. Liu 2008 et Luo 2013 sur les syllabes non-finales vs finale en CM). 7 apprenants réalisent [õ] comme une séquence $\mathrm{VN}$ [on] : « environ » [ауviвор], 12 donne la

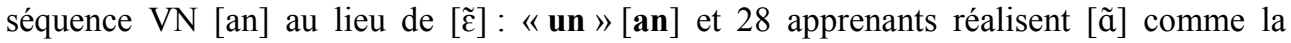
séquence VN [ay] : « France » [fqays].

\section{INFLUENCES DES L1 ET L2}

Dans $\S 3$ nous avons recensé toutes les voyelles du français réalisées par les apprenants chinois de notre corpus. Certaines stratégies d'adaptation peuvent facilement être identifié comme dominées par la L1 ou la L2, mais d'autres ont besoin d'une analyse plus minutieuse.

\subsection{Processus dominés par la L1}

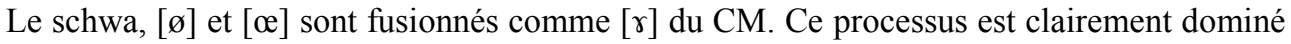
par la L1 car [r] est présent en CM mais absent en anglais.

Les séquences VN sont aussi classées dans cette catégorie. Elles remplacent les voyelles nasales cibles pour donner [on], [an] et [ay]. Ces VN séquences sont celles du CM et non pas celles de l'anglais comme [э๊] (song), [æn] (land) ou [æy] (hang).

\subsection{Processus dominés par la L2}

Le premier processus typique dominé par la L2 anglais est la prononciation relâchée des voyelles tendues : $[\mathrm{I}],[\mho]$ et $[æ]$ au lieu de [i], [u] et [a], parce que les voyelles relâchées n'existent pas en CM. Les apprenants préfèrent ici les relâchées de la L2 aux voyelles tendues existantes dans leur L1, ce qui montre que l'influence de la L2 pourrait être plus forte que celle de la L1.

Une hypothèse intéressante qu'on peut avancer est liée au transfert positif du lexique ayant comme effet de transfert négatif en phonétique. Pour [i] par exemple, dans « environ » [aјviкоn], notre apprenant réalise clairement $<\mathrm{i}>$ comme [i] tendu, il n'y a pas

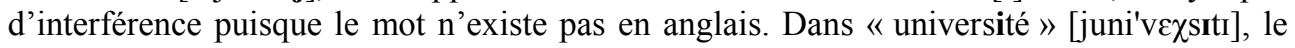
transfert de la L2 sur la L3 est positif en lexique car le mot est tout de suite reconnaissable et ne forme pas de faux-ami, mais en phonétique, le transfert devient négatif car le $<\mathrm{i}>$ est réalisé relâché comme en anglais.

Le deuxième processus est la réalisation [ju] de la monophtongue [y]. [ju] est une diphtongue de l'anglais alors qu'en $\mathrm{CM}$, même si [j] et [u] existent seuls, leur combinaison $\mathrm{j}+\mathrm{u}$ est mal formée comme séquence.

\subsection{Diphtongaison : L1 ou L2 ?}

Les voyelles moyennes $[\mathrm{e}],[\mathrm{o}],[\varepsilon]$ et [o] sont diphtonguées par une partie considérable des apprenants dans le corpus, pourtant, diphtongues existent en CM et en anglais.

Afin de déterminer si cette diphtongaison est due au CM et à l'anglais, nous recourons à Lindau et als (1990), étude phonétique comparative sur les diphtongues, qui montre que la différence majeure entre les diphtongues [ai] et [au] chinoises et anglaises consiste en leur durée de transition. Celle des diphtongues anglaises est nettement plus longue que celle des diphtongues chinoises. Pour cette raison, nous proposons que la 
diphtongaison [ai] et [au] des monophtongues est due à la L1, puisque les occurrences de ces diphtongues chez tous les apprenants ayant cette réalisation témoignent une durée de transition relativement courte.

Quant à la diphtongue [ou], elle est due également à la L1 parce que la diphtongue anglaise proche est [əซ] plutôt que [ou].

Enfin, la diphtongue [ei] est plus ambiguë. Phonétiquement, il y a peu de différence entre les diphtongues chinoise et anglaise notées [ei]. Toutefois, comme [ou] est à considérer comme dominé par la L1, sa contrepartie antérieure [ei] le devrait aussi, faute d'autres indices à ce stade.

\section{RESULTATS ET INTERPRETATION}

Les statistiques des observations dans $\S 3$ donnent le tableau suivant. Rappelons que le nombre total d'apprenants est de 44 .

\begin{tabular}{|c|c|c|c|c|c|c|c|c|c|c|c|c|c|c|}
\hline Voyelle & $\mathrm{i}$ & $\mathrm{y}$ & $\mathrm{u}$ & $\mathrm{e}$ & $\varnothing$ & o & $\varepsilon$ & $œ$ & 0 & $a$ & $\partial$ & $\overline{\tilde{\varepsilon}}$ & $\overline{\mathrm{o}}$ & $\overline{\mathrm{a}}$ \\
\hline $\begin{array}{l}\text { dominée } \\
\text { par la L1 }\end{array}$ & & & & 6 & 20 & 16 & 28 & 17 & 10 & & 31 & 7 & 12 & 28 \\
\hline$\%$ & & & & $14 \%$ & $45 \%$ & $36 \%$ & $64 \%$ & $39 \%$ & $23 \%$ & & $70 \%$ & $16 \%$ & $27 \%$ & $64 \%$ \\
\hline $\begin{array}{l}\text { dominée } \\
\text { par la L2 }\end{array}$ & 14 & 5 & 4 & & & & 2 & & & 9 & & & & \\
\hline$\%$ & $32 \%$ & $11 \%$ & $9 \%$ & & & & $5 \%$ & & & $20 \%$ & & & & \\
\hline
\end{tabular}

Pour certaines voyelles du français, des apprenants chinois préfèrent une adaptation aux sons existant en CM, alors que pour d'autres, ils cherchent plutôt des sons similaires en anglais.

Les voyelles hautes tendues, bien que présentes en CM, sont réalisées relâchées comme en anglais par certains apprenants. Les voyelles moyennes sont marquées par une forte tendance de diphtongaison. La voyelle basse est réalisée comme [æ] de l'anglais par quelques apprenants, malgré la présence de [a] tendu en CM. Les voyelles nasales ont une forte tendance à être réalisées comme des séquences $\mathrm{VN}$.

Comme suggère Cuet (2011), les apprenants chinois pensent souvent que l'anglais serait plus proche du français et ont tendance à se servir de l'anglais pour aider à apprendre le français. Cela expliquerait pourquoi une partie des apprenants sont sourds à des sons français qui pourtant existent phonétiquement en $\mathrm{CM}$, au profit des sons en anglais. Plus concrètement, nos observations préliminaires semblent lier le transfert positif en lexique à un transfert négatif en phonétique, comme par exemple le cas des voyelles relâchées.

Cet article se contente d'observer des stratégies d'adaptation détaillées de chaque voyelle du français dans un corpus constitué d'apprenants aux profils homogènes. Des études ultérieures doivent être effectuées en prenant compte du lexique, de la phonotactique, de la structure syllabique mais aussi de la prosodie.

\section{References}


Bradel, C. (2006). La connaissance d'une langue étrangère romane favorise-t-elle l'acquisition d'une autre langue romane ? Influences translinguistiques dans la syntaxe en L3. Acquisition et interaction en langue étrangère, 24, 149-179.

Cuet, C. (2008). Une didactique du compromis face aux éditeurs et professeurs chinois : concilier deux approches de l'enseignement des langues et innover dans l'élaboration de manuels. Cahiers de l'ACEDLE, 5(1), 151-166.

Cuet, C. (2009). Enseignement plurilingue en Chine. Une voie pour la recherche ? dans Forlot, G. ed., L'anglais et le plurilinguisme. Pour une didactique des contacts et des passerelles linguistiques, 117-140. Paris : L'Harmattan.

Cuet, C. (2009). Elaboration d'un manuel pour l'enseignement du français langue étrangère en Chine : le poids de la norme linguistique. Dans Quel français enseigner : La question de la norme dans l'apprentissage/enseignement, 345-360. Paris : Editions de l'Ecole Polytechnique.

Cuet, C. (2011). Enseigner le français en Chine, méthodologies nouvelles, perspectives. Synergies Chine, 6, 95-103.

Duanmu, S. (2007). The phonology of standard Chinese. Oxford University Press.

Gao, Yujuan \& Feng Shi (2006). Zhongguo xuesheng fayu yuanyin xuexi zhong muyu qianyi de shiyan yanjiu [Etudes expérimentale du transfert de la langue maternelle dans l'apprentissage du français chez les étudiants chinois]. Foreign languages and their teaching. 2006(4), 18-20.

Gut, U. (2010). Cross-linguistic influence in L3 phonological acquisition. International Journal of Multilingualism, 7(1), 19-38.

Hammarberg, B. (2001). Roles of L1 and L2 in L3 production and acquisition. In J. Cenoz, B. Hufeisen \& U. Jessner eds. Cross-linguistic influence in third language acquisition: Psycholinguistic perspectives, 21-41.

He, W. (2004). Dui yi hanyu wei muyu de yingyu xuexizhe shiyong yingshi fayin yu meishi fayin pianhao de diaocha [Enquête des préférences de prononciations britannique ou américaines de l'anglais chez les natifs du chinois]. Mémoire de master. Guangdong University of Foreign Studies.

Huang, Y., Delvaux, V., Li, J., Huet, K., Piccaluga, M., Zhang, G. \& Harmegnies, B. (2018). Une étude de la prononciation du français par des apprenants sinophones. Phonologie du français contemporain: usages, variétés, structure. https:/www.projet-pfc.net/wpcontent/uploads/2019/03/2018 Huang et al.pdf, consulté le 04/05/2020.

Landercy, A. \& Renard, R. (1977). Elements de phonétique. Bruxelle : Didier.

Landron, S., Gao, J. Chang, Y., et Tian, Y. (2016). Les sinophones. Dans S. Detey, I. Racine, Y. Kawaguchi, \& J. Eychenne (éds.), La prononciation du français dans le monde : du natif à l'apprenant. Paris : CLE International

Léon, P. (2007). Phonétisme et prononciation du français. Paris : Armand Colin.

Li, J., Yin Y. \& Pu Z. (2018). API et enseignement de la prononciation du français en Chine : médiation ou interférence ? Congrès mondial de la linguistique française, Mons, July 9-13.

Lindau, M., Norlin, K., \& Svantesson J.-O. (1990). Some Cross-linguistic Differences in Diphthongs. Journal of the International Phonetic Association 20(1), 10-14.

Liu, T.-h. (2008). Marque, registre et contour dans les systèmes tonals en chinois. Thèse de doctorat. Université Paris VIII.

Llama, R., Cardoso, W., \& Collins, L. (2010). The influence of language distance and language status on the acquisition of L3 phonology. International Journal of Multilingualism, 7(1), 39-57.

Luo, X. (2013). Vers une phonologie CVCV du chinois. Thèse de doctorat. Université d'Orléans.

Mei, Y. (2003). Zhongxiaoxue yingyu jiaoxue zhong meishi yingyu he yingshi yingyu de qufen he zhangwo [Distinction et maîtrise de l'anglais américain et de l'anglais britannique dans 
l'enseignement élémentaire et secondaire]. Jichu jiaoyu waiyu jiaoxue yanjiu [Enseignement et recherche des langues étrangères dans l'éducation élémentaire et secondaire]. 2003(9), 31-33.

Meillet, A. (1995). Pour un manuel de linguistique générale. Rome: Atti della accademia nationale dei lincei.

Martin, A. (2002). Convenance et résistance en F.L.E. (français langue étrangère) le cas des étudiants chinois. Verbum 4(1), 105-115.

Pöchtrager, M. \& Živanović, S. (2010). GP 2 and Putonghua too. Acta Linguistica Hungarica, 57(4), 357-380.

Rimpault, D. (2018). Interphonologie et remédiation phonétique en Français Langue Étrangère : Enjeux didactiques pour l'enseignement de la prononciation à des apprenants chinois. Mémoire de master. Université Toulouse Jean Jaurès.

Scheer, T. (1999). Aspects de l'alternance schwa-zéro à la lumière de CVCV. Recherches Linguistiques de Vincennes 28, 87-114.

Wang, H. \& Ma, J. (2005). Xiangsi zhong de busi: qianxi shuangyu zhuanye fayu jiaoxue zhong de yingyu ganrao wenti [Différences et similitudes : de l'interférence de l'anglais dans l'enseignement du français en spécialités bilingues]. Bulletin de Xi'an University of Foreign Studies, 2005(2), 31-34.

Wang, M. (2008). Yingfa yuyin duibi zai fayu jiaoxue zhong de yingyong [Application de la comparaison anglais-français dans l'enseignement du français]. Exam Weekly, 2008(5), 73-74.

Wlosowicz, T. M. (2010). Le transfert et les interférences entre L1, L2 et L3 dans la production des cognates aux terminaisons différentes. Synergies Espagne, 3, 159-170.

Xu, C. \& Zhang, J. (2011). Yingyu dui fayu yuyin qianyi ji dui fayu yuyin jiaoxue de qishi [Révélations de l'anglais pour le transfert de la phonétique du français et son enseignement]. Bulletin de l'Université Normale de Hebei, Science de l'Education. 2011(12).

Zhou, X. (2006). Fahui yingyu zai fayu jiaoxue zhong de fuzhu zuoyong [Le rôle de l'anglais dans l'enseignement du français]. Bulletin de Wuhan Institute of Science and Technology, 2006(12), $170-173$.

\footnotetext{
${ }^{\mathrm{i}}$ Les langues chinoises constituent une famille de langues qui se déclinent en 8 à 10 groupes selon les auteurs. Pour des raisons de la politique linguistique, elles sont appelées dialectes malgré l'intercompréhension impossible.

${ }^{i i}$ Quand on a affaire à la séquence [jø] (ex. mieux, Monsieur), [jø] est adapté à la diphtongue [ju] de l'anglais comme un atome par 5 apprenants.
} 